\title{
Hybrid Metaheuristic Aided Energy Efficient Cluster Head Selection in Wireless Sensor Network
}

\author{
Turki Ali Alghamdi \\ Department of Computer Science, College of Computer and \\ Information Systems, Umm Al-Qura University, Makkah, Saudi Arabia
}

\begin{abstract}
Clustering is one of the significant techniques for expanding the lifetime of networks in wireless sensor networks (WSNs). It entails combining of sensor nodes (SNs) into clusters and electing cluster heads (CHs) for each and every cluster. $\mathrm{CH}$ collects the information from particular cluster nodes and passes the cumulative data to the base station (BS). However, the most important requirement in WSN is to choose a suitable $\mathrm{CH}$ with an increased network life span. This work introduces a new CHS model in WSN. The optimal $\mathrm{CH}$ is elected by a new hybridized model termed as "Lion Updated Dragonfly Algorithm (LU-DA) that hybrid the concepts of Dragonfly Algorithm (DA) and Lion Algorithm (LA)". Moreover, the optimal selection of CH is done depending upon constraints like "energy, delay, distance, security (risk) and trust (direct and indirect trust)". This optimal CH ensures the network lifetime enhancement. At last, the superiority of the developed approach is proved on varied measures like energy and alive node analysis. Accordingly, the proposed model has accomplished higher energy of 0.55 at $1^{\text {st }}$ round, whereas at the $2000^{\text {th }}$ round, the normalized energy value has been dropped to 0.1 .
\end{abstract}

Keywords-Cluster head; security; trust; dragonfly algorithm; LU-DA model

Nomenclature

\begin{tabular}{|l|l|}
\hline Abbreviation & Description \\
\hline ACO & Ant Colony Optimization \\
\hline APTEEN & $\begin{array}{l}\text { Adaptive Threshold sensitive Energy Efficient sensor } \\
\text { Network }\end{array}$ \\
\hline BS & Base Station \\
\hline BOA & Butterfly Optimization Algorithm \\
\hline CH & Cluster Head \\
\hline CHS & Cluster Head Selection \\
\hline DA & Dragonfly Algorithm \\
\hline EE & Energy Efficiency \\
\hline FF & Firefly \\
\hline FCM & Fuzzy C-Means \\
\hline FPU-DA & fire fly replaced position update in dragonfly \\
\hline GECR & $\begin{array}{l}\text { Genetic Algorithm-based Energy-Efficient Clustering and } \\
\text { Routing }\end{array}$ \\
\hline GWO & grey wolf optimizer \\
\hline GA & Genetic Algorithm \\
\hline HSO & Harmony Search Optimization \\
\hline HML & Hierarchical Maximum Likelihood \\
\hline
\end{tabular}

\begin{tabular}{|l|l|}
\hline KFCM & kernel fuzzy C-means \\
\hline LEACH & Low-Energy Adaptive Clustering Hierarchy \\
\hline LU-DA & Lion Updated Dragonfly Algorithm \\
\hline LA & Lion Algorithm \\
\hline MOFPL & Multi-objective fractional particle lion algorithm \\
\hline NAN & Number of Alive Node \\
\hline OWSN & Optical WSN \\
\hline PSO & Particle Swarm optimization \\
\hline PEGASIS & Power-Efficient Gathering in Sensor Information Systems \\
\hline QoS & Quality of Services \\
\hline QCM2R & QoS-aware cross-layered multichannel multisink routing \\
\hline SNs & Sensor Nodes \\
\hline TEEN & Threshold sensitive Energy Efficient sensor Network \\
\hline WSN & Wireless Sensor Network \\
\hline
\end{tabular}

\section{INTRODUCTION}

WSN [1] [2] involves various sensors connected to the wireless medium. The sensed data from SNs is typically forward to $\mathrm{BS}$, where the data is collected, analyzed and performed certain actions accordingly [3]. The WSN is deployed in various applications like weather monitoring [4], meteorological data collection [5], and field surveillance, transportation, and health-care] [6] [7]. However, the nodes in WSN don't have any storage devices and facilities of researchable batteries [8] [9]. Though, it should support any system with effective power consumption [10] [11].

Clustering is a renowned procedure for effective data transmission with respect to energy and power utilization. Clustering involves dividing of SNs into different clusters [12] [13] [14]. All clusters in networks have distinctive CHs [15] [16], which is responsible to transfer information to other SNs in its cluster. Moreover, the communication to BS is carried out only through this $\mathrm{CH}$. In this scenario, key role is to opt the optimal $\mathrm{CH}$ by concerning on lesser delay and low consumption of energy [17] [18]. Thereby, creating a cluster with aggregation and data fusion models, there is energy in network based on the data transmitted to BS [19] [20].

Thereby, the cluster-oriented models also engaged in facilitating the extension of network lifetime [21]. The frequently deployed algorithms include APTEEN, TEEN, LEACH, PEGASIS, and FCM. Further, "LEACH is the cluster-based algorithm that operates in the distributed 
manner, which elects the $\mathrm{CH}$ depending on the predetermined probability" [22].

Various cluster-oriented models have been introduced so far, which is based on meta-heuristic algorithms. However, the algorithms possess some common challenges such as high convergence, local search issues in FF, and high cost. Moreover, there is a prerequisite of standard optimizations and need consideration on constraints, namely security and trust [23]. So in order to solve the above mentioned issues, this paper introduces a new CHS model in WSN.

The foremost contribution is listed here:

- The selection of optimal $\mathrm{CH}$ depends on certain constraints such as energy consumption, trust, security, delay and distance.

- Proposes a Lion Updated Dragonfly Algorithm for optimal $\mathrm{CH}$ selection, which integrates the concepts of DA as well as LA models.

The organization of the paper is as follows: Section II reviews CHS models. Section III elaborates the adopted energy aware clustering model in WSN. Section IV depicts the optimal CHS: objective model. Section V explains Lion Updated Dragonfly Algorithm for optimal CHS. The resultants and conclusions are briefed in Section VI and Section VII.

\section{LITERATURE SURVEY}

\section{A. Related Work}

In 2020, Turki et al. [24] suggested a new clustering model with optimal CHS that considered 4 most important criterions such as security, delay, energy, and distance. Furthermore, for electing the optimum $\mathrm{CHs}$, this work proposed a novel algorithm named as FF-PUD. At last, the performances of developed scheme were performed by evaluating it over other schemes regarding risk, alive nodes, energy and delay.

In 2020, Prachi et al. [25] have employed BOA for choosing an optimal $\mathrm{CH}$ from nodes. Accordingly, the developed work aims on lessening the energy usage and for maximizing the life span of network. The path among the $\mathrm{CH}$ and BS was determined using ACO and it selected optimal routes depending on node degree, residual energy and distance. At the end, the supremacy of adopted work was proved regarding energy consumption, alive and dead nodes.

In 2019, Reeta and Dinesh [26] have designed a multiobjective model that dependents on distance, traffic rates, energy, cluster densities and delay. Here, energy based routing was carried out based on MOFPL scheme. The implemented model determined the optimum $\mathrm{CH}$ from several nodes in WSN. Consequently, the optimal routes were introduced depending on the adopted multi-objective function. Furthermore, effective CHS with high network energy was accomplished by the designed model.

In 2020, Augustine and Ananth [27] have presented an enhanced framework for CHS based on Taylor KFCM that was modified from the KFCM approach in the Taylor series.
The introduced model has chosen the $\mathrm{CH}$ by means of "acceptability factor" that was evaluated by the trust, distance, and energy. Further, the advantage of the proposed system was proved in terms of highest energy and high trust.

In 2019, Goswami et al. [28] introduced a cluster-based model by deploying HML and FF model in OWSN for improving the EE and minimizing the costs. Here, the issues in FF model were prevailed over by integrating the theory of HML with it. Furthermore, the distribution of power in nodes was carried out precisely via maximum likelihood property of HML. Finally, the resultants have shown the betterment of presented scheme regarding $\mathrm{EE}$ and cost function.

In 2019, Jain and Toor [29] offered a new framework for diverse WSN by considering "MEACBM routing protocol". Accordingly, optimal election of CHs takes place; particularly, the SNs with higher energy were preferred as $\mathrm{CH}$. This model has minimized the energy utilization of SNs while conveying data to BS. The analysis resultants have revealed the enhancement regarding the $\mathrm{CH}$ count, network lifetime, throughput and dead node count.

In 2019, Daneshvar et al. [30] have offered a new clustering scheme, which selected CHs by means of GWO. For selecting $\mathrm{CHs}$, the solutions were optimized depending on remaining energy of every node and predicted energy utilization. In addition, for improving the EE, the presented model deployed the similar clustering in numerous successive rounds. This allowed the framework to accumulate the energy, which was necessary for reforming the clustering. Eventually, the outcomes demonstrated that the designed model has ensured effective network lifetime.

In 2018, Tianshu et al. [31] suggested a routing scheme depending upon GECR and GA for expanding the lifetime of networks and improving EE. In addition, while modelling the objective function, the "load balancing factor" was taken into account that balanced the energy usage among SNs. The simulated results have exposed the supremacy of the adopted method with lower variance and improved EE.

\section{B. Problem Formulation}

Table I makes a review of existing cluster-based energyaware CHS models in WSN. Numerous methods have been focused on energy-aware CHS models in WSN. But still, the existing models like FF-PUD[24], BOA + ACO [25], MOFPL [26], Taylor KFCM model [27], FF [28] have some common problems like high convergence, local search issues in FF, high-cost efficiency, there is a need of standard optimizations and need consideration on constraints like security and trust.

\section{Objectives}

The main objectives of this paper are:

- To select an optimal CH depends on certain constraints such as energy consumption, trust, security, delay and distance.

- To propose an improved Algorithm for optimal $\mathrm{CH}$ selection for solving the optimization issues.

- And to improve the better convergence rate. 
TABLE I. REVIEWS ON TRADITIONAL ENERGY AWARE CHS MODELS IN WSN

\begin{tabular}{|c|c|c|c|}
\hline Authors & Techniques & Feature & Challenge \\
\hline Turki et al. [24] & FF-PUD & $\begin{array}{ll}* & \text { Minimal delay } \\
* & \text { High network energy }\end{array}$ & * Coverage issues are not deliberated. \\
\hline Prachi et al. [25] & $\mathrm{BOA}+\mathrm{ACO}$ & $\begin{array}{l}* \quad \text { Higher count of alive nodes } \\
\quad \text { Minimal energy consumption }\end{array}$ & * Should consider fault tolerance. \\
\hline Reeta and Dinesh [26] & MOFPL & $\begin{array}{l}* \quad \text { Less simulation time } \\
* \quad \text { Offers high network energy }\end{array}$ & $\begin{array}{l}* \quad \text { Resource management is not taken into account in this work. } \\
\text { Cost efficiency is not considered. }\end{array}$ \\
\hline $\begin{array}{l}\text { Augustine and Ananth } \\
\text { [27] }\end{array}$ & $\begin{array}{l}\text { Taylor KFCM } \\
\text { model }\end{array}$ & $\begin{array}{l}\text { High throughput and energy. } \\
\text { Minimal delay }\end{array}$ & $\begin{array}{l}\text { No consideration on real time experiments. } \\
\text { Standard optimizations are required for enhancing the CHS } \\
\text { performance. }\end{array}$ \\
\hline Goswami et al. [28] & $\mathrm{FF}$ & $\begin{array}{l}* \quad \text { Minimal cost function. } \\
\quad \text { Improved EE }\end{array}$ & FF suffers from local search issues. \\
\hline Toor and Jain [29] & MEACBM & $\begin{array}{l}\text { Minimized the consumption of } \\
\text { energy } \\
* \quad \text { Raises throughput and lifetime }\end{array}$ & * Needs consideration on scalability of SNs \\
\hline Daneshvar et al. [30] & GWO & $\begin{array}{l}* \quad \text { Balanced energy consumption } \\
\quad \text { Offers high life span for network }\end{array}$ & * Fault tolerance is not considered. \\
\hline Tianshu et al. [31] & GECR & $\begin{array}{l}\text { Better life span } \\
\quad \text { Optimal energy utilization }\end{array}$ & * More appropriate metaheuristic algorithms should be used. \\
\hline
\end{tabular}

\section{Proposed Energy Aware Clustering Model IN WSN}

\section{A. Network Model}

Assume $M_{n}$ sensor nodes that are randomly deployed in appliance area. Consequently, the clustering process is done by merging the SNs. During clustering, the nodes forms clusters, wherein a $\mathrm{CH}$ is elected and the total count of $\mathrm{CH}$ is delineated by $\mathrm{CH}_{n}$. Thus, the distances amongst nodes and $\mathrm{CHs}$ have to be reduced.

The most important task of WSN is to transfer the information among nodes. Here, the identification of shorter paths is required to enhance the data transmission. Moreover, the energy consumption of node also acts as the most role while transmitting the data. Particularly, a node requires more energy for transmitting massive data. In the clustering based strategy, the $\mathrm{CH}$ is responsible for transmitting more data with less energy consumption. However, the security is more crucial for minimizing the overhead and attacks. The architectural depiction of adopted model with varied SNs is illustrated in Fig. 1.

\section{B. Distance Model}

In the network, a $\mathrm{CH}$ is chosen only if the distance between $\mathrm{CH}$ and nodes is minimal. If distance among $\mathrm{CH}$ and nodes are higher than distances amid node and BS, the data are transmitted directly to BS by node. By deploying distance matrix $\operatorname{Di}\left(g^{*} w\right)$, the SNs gets clustered with selected $\mathrm{CH}$ as exposed in Eq. (1), wherein, $e_{M_{C H}}$ signifies Euclidean distance amid $M_{C H}$ and normal node position, and $z_{1}, z_{2}, \ldots z_{n}$ signifies SNs. Assume 2 SNs $q$ and $d$, and positions be $x$ and $y$. The Euclidean distances amongst 2 nodes are revealed in Eq. (2). In Eq. (1), element $e_{M_{C H 2}, z_{1}}$ occupies initial column matrix with minimal distance [24].

$$
D i(g * w)=\left[\begin{array}{l}
e_{M_{C H 1}, z_{1}} e_{M_{C H 1}, z_{2}} \ldots \ldots . . e_{M_{C H 1}, z_{n}} \\
e_{M_{C H 2}, z_{1}} e_{M_{C H 2}, z_{2}} \ldots \ldots . . e_{M_{C H 2}, z_{n}} \\
\vdots \\
\vdots \\
e_{M_{C H m}, z_{1}} e_{M_{C H m}, z_{2}} \ldots \ldots . . e_{M_{C H m}, z_{n}}
\end{array}\right]
$$

$e_{q, d}=\sqrt{\left(q_{x}-d_{x}\right)^{2}+\left(q_{y}-d_{y}\right)^{2}}$

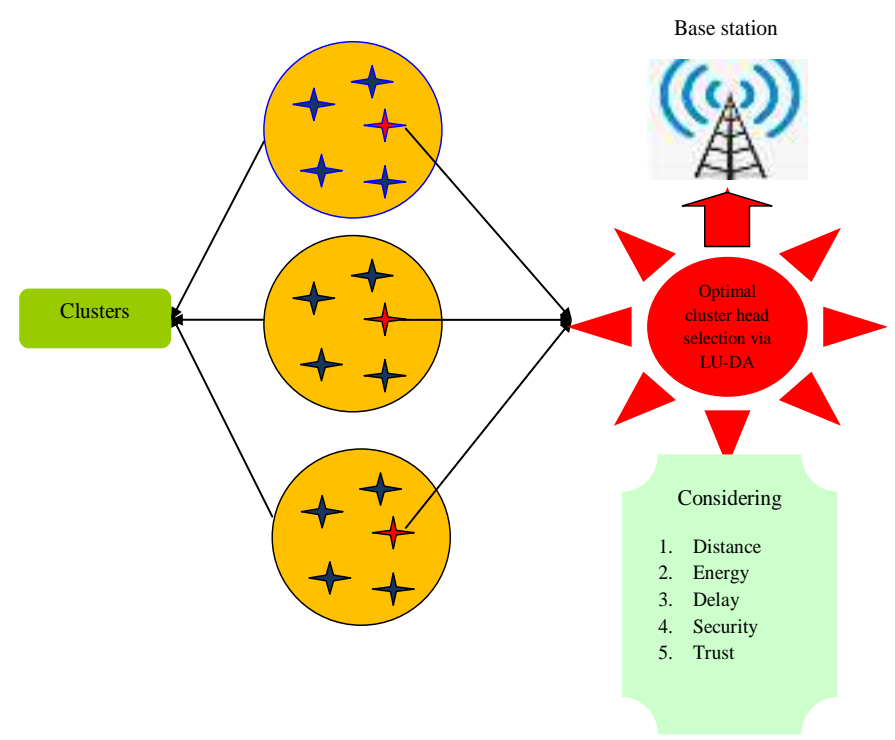

Fig. 1. Architecture of Proposed CHS Model. 
Further, the time slots are assigned by $M_{C H}$ to every node during data transmission. Here, $M_{C H}$ collects data from all SNs in clusters. After data gathering, $M_{C H}$ passes the specified data to BS.

\section{Energy Model}

Energy utilization is a foremost characteristic in WSNs. Actually, additional energy is crucial for conveying data to BS from every SNs. Thereby, the energy model for transmitting data is exposed in Eq. (3), wherein, " $E_{\text {ete }}$ symbolizes the electronic energy as given in Eq. (4), wherein $E_{\text {agg }}$ refers to the energy utilization during data collection and $E_{T X}(M: e)$ signifies the energy necessary for transferring $M$ bytes of packets at distance $e$ ". Eq. (5) shows the essential energy for passing $M$ bytes of packets. Eq. (6) shows the "amplification energy and $E_{p r}$ refers to power amplifier energy and $E_{f r}$ refers to energy required for deploying free space technique" [24].

$$
\begin{aligned}
& E_{T X}(M: e)=\left\{\begin{array}{l}
E_{\text {ete }} * M+E_{f r} * M * e^{2}, \text { if } e<e_{0} \\
E_{\text {ete }} * M+E_{p r} * M * e^{2}, \text { if } e \geq e_{0}
\end{array}\right. \\
& E_{\text {ete }}=E_{T X}+E_{a g g} \\
& E_{R X}(M: e)=E_{\text {ete }} M \\
& E_{a g g}=E_{f r} e^{2} \\
& e_{0}=\sqrt{\frac{E_{f r}}{E_{p r}}}
\end{aligned}
$$

The whole energy of network is given in Eq. (8), wherein $E_{1}$ symbolizes the energy at idle state and $E_{S T}$ symbolizes energy at sensing time.

$$
E_{\text {total }}=E_{S T}+E_{1}+E_{R X}+E_{T X}
$$

\section{Security Model}

The risky mode, $\gamma$-risky mode and security mode are the factors in security model that are explained below.

"Risky mode: This mode selects an existing $\mathrm{CH}$ for facilitating an optimal CHS, for which it takes all the risks. Thus, this mode is considered as an insistent mode while choosing $\mathrm{CH}$ [24].

$\gamma$-risky mode: The $\mathrm{CH}$ which could tolerate the utmost $\gamma$-risk are elected based upon $\gamma$-risky mode. Accordingly, $\gamma$ signify the probability measure with values, $\gamma=0$ and $\gamma=1$ (i.e., $100 \%$ ) similar to security and risky mode.

Security mode: This mode prefers the $\mathrm{CH}$ that fulfills the needs of security. In Eq. (1), $s_{r}$ and $s_{d}$ denotes the security rank and security needs associated with CHS. If $s_{d} \leq s_{r}$, the node are considered as $\mathrm{CH}^{\prime}$.
The probability of security constraints is shown in Eq. (9). Further, "if the chosen $\mathrm{CH}$ achieves the state $s_{d}>s_{r}$ the risk should be less than $50 \%$. If the condition is $0<s_{d}-s_{r} \leq 1$, the selection process would be implemented, and if the state is $1<s_{d}-s_{r} \leq 2$, there will be a delay in the selection process. Still, the CHS process could not be completed, and the corresponding function should be continued for the state $2<s_{d}-s_{r} \leq 5 "$.

$$
g_{\text {risk }}=\left\{\begin{array}{ccc}
0 & \text { if } & s_{d}-s_{r} \leq 0 \\
1-e^{\frac{\left(s_{d}-s_{r}\right)}{2}} & \text { if } & 0<s_{d}-s_{r} \leq 1 \\
1-e^{\frac{3\left(s_{d}-s_{r}\right)}{2}} & \text { if } & 1<s_{d}-s_{r} \leq 2 \\
1 & \text { if } & 2<s_{d}-s_{r} \leq 5
\end{array}\right.
$$

\section{E. Trust}

"Trust is the degree of reliability about other node for performing certain action by keeping track of all past transaction or interactions with nodes by direct or indirect observation. Trust can also be defined as the level of confidence that one node about other node to get assigned work done within some time". Trust includes direct as well as indirect trust. The final trust is computed by combining both indirect and direct trust values [32].

Direct trust: It is computed depending upon interaction of nodes. The distance and energy are regarded as trust measures and is evaluated as per Eq. (10), where $D T_{(A-G)}$ denotes the value of direct trust computed by $A$ and $G, E r$ denotes residual energy of node $G, d(\operatorname{node} A, \operatorname{node} G)$ refers to differentiation distance of node $A$ and $G$.

$$
D T_{(A-G)}=\frac{E r}{d(\text { node } A, \text { node } G)}
$$

Indirect trust: It is computed depending upon recommendation of nodes. It is the summation of trust values computed by other nodes and specified as in Eq. (11), wherein, $I D T_{(A-G)}$ refers to value of direct trust computed by $A$ and $G, D T(A-P)$ and $D T(P-G)$ refers to value of direct trust computed by $A, P$ as well as $P$ and $G$ in that order.

$$
I D T_{(A-G)}=\sum D T(A-P) \times D T(P-G)
$$

Further, the final trust is computed as in Eq. (12), wherein, $T_{(A-G)}$ refers to final trust $A$ on $G$ and $w$ refers to weight related with indirect and direct trusts.

$$
T_{(A-G)}=w D T_{(A-G)}+(1-w) I D T_{(A-G)}
$$




\section{Optimal Cluster Head Selection: ObJective MODEL}

This work aims to diminish the distance amid the chosen $\mathrm{CH}$ and $\mathrm{SN}$ and it aims to lessen the delay and risk while transferring the information. On the other hand, the energy, and trust have to be high for better transmission of data. The objective of developed model is delineated in Eq. (13), in which $\eta$ relies amid $0<\eta<1, o_{m}$ and $o_{n}$ are calculated as revealed in Eq. (14) and Eq. (15), respectively. The delay, energy, distance, security and trust are explained by $\varpi_{1}, \varpi_{2}$, $\varpi_{3}, \quad \varpi_{4} \quad, \varpi_{5} \quad$ and $\quad$ are represented as $\varpi_{1}+\varpi_{2}+\varpi_{3}+\varpi_{4}+\varpi_{5}=1$. In Eq. (15), $Z_{z}-A_{s}$ depicts distance amid normal node and sink.

$$
\begin{aligned}
& K_{n}=\eta o_{n}+(1-\eta) o_{m} \\
& o_{m}=\varpi_{1}{ }^{*} o_{i}^{d e l}+\varpi_{2} * o_{i}^{e n e}+\varpi_{3} * o_{i}{ }^{d i s}+\varpi_{4} * o_{i}{ }^{S e c}+\varpi_{5} * o_{i}^{T} \\
& o_{n}=\frac{1}{b} \sum_{z=1}^{b}\left\|Z_{z}-A_{s}\right\|
\end{aligned}
$$

The fitness function for distance is specified by Eq. (16), wherein, $o_{(m)}^{\text {dis }}$ signify packets passed between $\mathrm{SN}$ to $\mathrm{CH}$ and between $\mathrm{CH}$ to $\mathrm{BS} . o_{i}{ }^{\text {dis }}$ lies amongst $[0,1]$.

$$
\begin{aligned}
& o_{i}^{d i s}=\frac{o_{(m)}^{d i s}}{o_{(n)}^{d i s}} \\
& o_{(m)}^{d i s}=\sum_{z=1}^{M_{z}}\left[\left\|C H_{z}-A_{s}\right\|+\sum_{x=1}^{M_{x}}\left\|C H_{z}-Z_{z}\right\|\right] \\
& o_{(n)}^{d i s}=\sum_{z=1}^{M_{z}} \sum_{x=1}^{M_{x}}\left\|Z_{s}-Z_{x}\right\|
\end{aligned}
$$

$o_{(m)}^{d i s}$ and $o_{(n)}^{d i s}$ are modelled as in Eq. (17) and (18), here $Z_{z}$ symbolizes $\mathrm{SN}$ in $z^{\text {th }}$ cluster, $\mathrm{CH}_{z}$ symbolizes $\mathrm{CH}$ of $z^{\text {th }}$ cluster, the distance amid $\mathrm{BS}$ and $\mathrm{CH}$ is indicated as $\mathrm{CH}_{z}-A_{s}, \mathrm{CH}_{z}-\mathrm{Z}_{z}$ symbolizes distance among $\mathrm{CH}$ and $\mathrm{SN}$ and $Z_{z}-Z_{x}$ symbolizes distance among 2 SNs, $M_{z}$ and $M_{x}$ symbolizes node count devoid of considering $x^{\text {th }}$ and $z^{\text {th }}$ clusters.

The fitness function for energy $\left(o_{i}{ }^{\text {ene }}\right)$ is revealed in Eq. (19), here, $o_{(m)}^{\text {ene }}$ and $o_{(n)}^{\text {ene }}$ symbolizes high value of energy and larger $\mathrm{CH}$ count.

$$
o_{i}^{e n e}=\frac{o_{(m)}^{\text {ene }}}{o_{(n)}^{\text {ene }}}
$$

The fitness function for delay $\left(o_{i}^{d e l}\right)$ is revealed in Eq. (20) and it is measured for every $\mathrm{SN}$ in cluster. Thus, delay gets lessened if the count of SN in CH is minimal. In Eq. (20), $M_{n}$ symbolizes total node count, and numerator depicts the higher $\mathrm{CH}$ count.

$o_{i}^{d e l}=\frac{\max \left(\left\|C H_{z}-Z_{z}\right\|\right)_{z=1}^{M_{C H}}}{M_{n}}$

\section{PROPOSED LiON UPDATED DRAGONFLY AlgORITHM FOR OPTIMAL CLUSTER HEAD SELECTION}

\section{A. Solution Encoding}

The proposed work focuses on introducing an efficient CHS in WSN. In WSN, the optimum selection of $\mathrm{CH}$ is a versatile task and it is performed based upon criteria like, trust, security energy, delay and distance. The input provided to LU-DA algorithm is shown by Fig. 2.

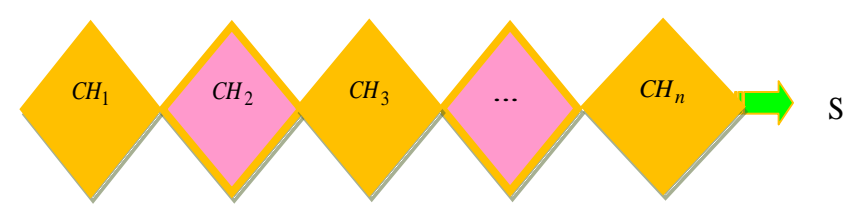

Fig. 2. Solution Encoding.

\section{B. Proposed LU-DA Algorithm}

Although the conventional DA [33] model encompasses a variety of enhancements; it suffers from specific limitations like, complexity in solving binary problems etc. Therefore, the theory of LA [34] is mingled with it to introduce a new model named as LU-DA. Hybridized optimization schemes are said to be more appropriate for specific search issues [23] [35] [36] [37]. The steps followed in the proposed LU-DA are as follows.

LU-DA model involve two stages such as: “(i) Exploration and (ii) Exploitation". The separation formula is modelled as exposed in Eq. (21), where, $S_{l}$ signifies $l^{\text {th }}$ closer individual's position, $S$ and $U$ symbolizes current position of individual and nearby individual's count.

$$
C_{i}=-\sum_{l=1}^{U}\left(S-S_{l}\right)
$$

Consequently, the alignment is evaluated as specified in Eq. (22), wherein, $Q_{l}$ denotes velocity of $l^{\text {th }}$ closer individual. The cohesion is modelled as in Eq. (23) and attraction to food is evaluated as in Eq. (24), wherein $S^{+}$ symbolizes position of food source and $S$ symbolizes present individual position.

$$
B_{i}=\frac{\sum_{l=1}^{U} Q_{l}}{U}
$$


$O_{i}=\frac{\sum_{l=1}^{U_{b}} S_{l}}{U_{b}}-S$

$F_{i}=S^{+}-S$

Distraction to enemies is indicated by Eq. (25), wherein, the enemy position is denoted as $S^{-}$.

$E n_{i}=S^{-}+S$

Eq. (26) shows the modelling of step vector $(\Delta S)$, wherein, " $v$ signifies separation weight, $C_{i}$ denotes the separation of $i^{\text {th }}$ individual, $O$ denotes the $i^{t h}$ individual cohesion, $c$ points out cohesion weight, $B_{i}$ and $F_{i}$ refers to the alignment and food resources of $i^{\text {th }}$ individual, $a$ signifies the alignment weight, $f$ corresponds to food factor, $b$ symbolizes enemy factor, $h$ signifies the inertia weight, $E n_{i}$ refers to enemy's position of $i^{\text {th }}$ individual and $i t$ points out iteration counter".

$\Delta S(i t+1)=\left(v C_{i}+a B_{i}+c O_{i}+f F_{i}+b E n_{i}\right)+h \Delta S(i t)$

As per LU-DA model, if random integer $r a \leq \Delta S_{t+1}$ and if $\Delta S_{t+1} \leq 0$, the position vector ( $S$ ) is computed as in Eq. (27), wherein, it signifies current iteration.

$S(i t+1)=S(i t)+\Delta S(i t+1)$

On the other hand, if $r a \leq \Delta S_{t+1}$ and if $\Delta S_{t+1}>0$, the position gets updated based on proposed female lion update as shown in Eq. (28), where, $\nabla_{d}$ is evaluated as in Eq. (29), levy $(\beta)$ denotes levy flight. $S_{d}^{f e m+}$ signifies $k^{\text {th }}$ vector elements of $S^{\text {fem+ }}, \nabla$ refers to update function of female, $k$ denotes arbitrary integer and $\breve{r}_{2}, \breve{r}_{1}$ signifies arbitrary integer among $[0,1]$.

$S_{d}^{f e m+}=\min \left[S_{d}^{\max }, \max \left(S_{d}^{\min }, \nabla_{d}\right)\right]+\operatorname{levy}(\beta)$

$\nabla_{d}=\left[S_{d}^{f e m}+\left(0.1 \breve{r}_{2}-0.05\right)\left(S_{d}^{m a l}-\breve{r}_{1} S_{d}^{f e m}\right)\right]$

Else if, $r a>\Delta S_{t+1}$, the position gets updated as in Eq. (30).

$S_{t+1}=S_{t}$
Algorithm 1 shows the pseudo code of presented LU-DA scheme.

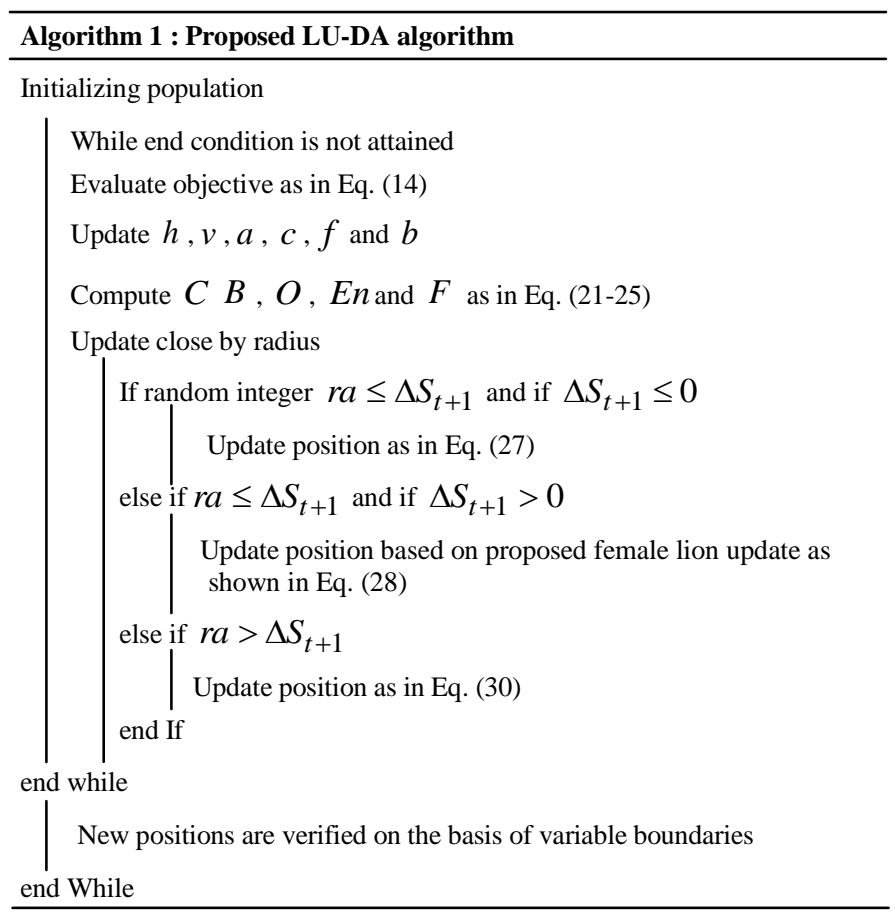

\section{RESULT AND DISCUSSION}

\section{A. Simulation Setup}

The adopted LU-DA based CHS in WSN was simulated in MATLAB. The analysis was held by evaluating the alive node count for varied number of round that ranges from 0 t0 2000. Further, $\log$ of alive node count was analysed for varied distance that range from $0,20,40,60$ and 80 . In addition, cost analysis was done for varied iterations that range from $0,2,4$, 6, 8 and 10. Also, the proposed model was computed over extant approaches such as FF [8], GWO [9], LA [34], DA [23] and FPU-DA [24] and the outcomes were examined in terms of statistical analysis. The simulation parameters in this work are summarized in Table II.

TABLE II. SIMULATION PARAMETERS

\begin{tabular}{|l|l|}
\hline Parameters & Values \\
\hline “Initial nodal energy & $0.5 \mathrm{~J}$ \\
\hline Fraction of super nodes amidst advanced nodes & 0.6 \\
\hline Network area & $100 \times 100$ \\
\hline Energy factor of super node & 3 \\
\hline Fraction of advanced sensor nodes amidst normal nodes & 0.4 \\
\hline Total node count & 100 \\
\hline Energy dispersed per bit & $100 \mathrm{~nJ} / \mathrm{bit}$ \\
\hline Data packet aggregation energy & $5 \mathrm{~nJ} / \mathrm{bit} / \mathrm{message"}$ \\
\hline
\end{tabular}




\section{B. Analysis on Alive Nodes}

The Analysis on NAN of suggested LU-DA scheme over FF, GWO, LA, DA and FPU-DA models is specified in Fig. 3(a), whereas, the log of NAN analysis is shown in Fig. 3(b). The Analysis on NAN is done for 2000 rounds, while, $\log$ of NAN is performed for varied distance that range from $0,20,40,60$ and 80. In fact, the transmission of data and clustering operations continues for several rounds up to the death of every node. Thereby, the NAN in cluster gets reduced in every cluster. Thus, the NAN reduces with raise in rounds. Till $700^{\text {th }}$ round, the NAN for conventional and proposed models is 100 , and it is gradually reduced as the round gets improved. Nevertheless, at $2000^{\text {th }}$ round, the adopted scheme reveals higher NAN than extant models, thus guarantying the enhanced performance of adopted scheme. Particularly, within $75 \%$ variation in rounds (i.e. from $500^{\text {th }}$ round to $2000^{\text {th }}$ round), the NAN using presented technique has dropped from 100 to 40 . On the other hand, for similar variation (75\%) in rounds, the NAN using conventional GWO has dropped from 100 to 19 . Thus, the analysis established the enhanced efficacy of LU-DA method with the subsistence of more NAN.

\section{Analysis on Normalized Energy}

Fig. 4 describes the examination on normalized energy attained using suggested LU-DA model over traditional models for varied number of rounds that ranges from 0 to 2000. The normalized energy is portrayed depending upon the residual network energy and it have to be high for better system performance. In Fig. 4, the network energy seems to be higher at initial rounds; however, with increase in rounds, the energy starts lessening steadily for both adopted as well as compared extant schemes. Especially, from Fig. 4, the presented model has attained higher energy of 0.55 at $1^{\text {st }}$ round, while at $2000^{\text {th }}$ round; the normalized energy value has been dropped to 0.1. However, the adopted model has accomplished a higher energy even at $2000^{\text {th }}$ round, when distinguished over FF, GWO, LA, DA and FPU-DA models. Thus, the capable performance of developed model is confirmed.

\section{Convergence Analysis}

Fig. 5 describes the convergence analysis of the adopted model over conventional approaches regarding cost. Here, Analysis is performed for a varied number of iterations that ranges from $0,2,4,6,8$ and 10 . On noticing the analysis resultants, the developed LU-DA has a negligible cost for all iterations over conventional approaches. Predominantly, on noticing cost values from Fig. 5, the adopted scheme has attained reduced cost value (0.06) from iteration 3 to 10 . At the initial iterations (from 1 to 3 ), the cost of developed model has accomplished a comparatively higher value, while at further iterations; the developed model has converged to a minimal cost value. Especially, at iteration 2, the adopted model is only $60 \%$ enhanced than extant FF model, while at iteration 10, the adopted model is $62.5 \%$ enhanced than FF model. Thus, the overall assessment shows the impact of the developed LU-DA on better convergence results with increase in iterations.

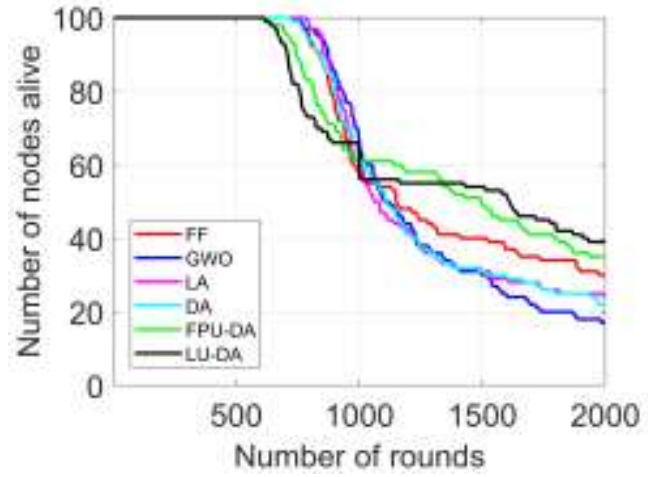

(a)

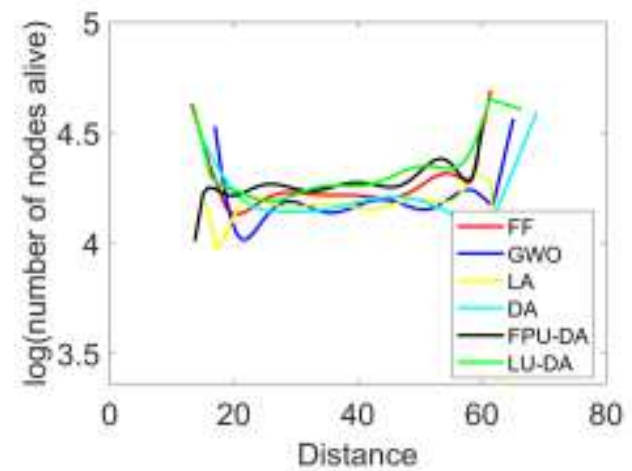

(b)

Fig. 3. NAN Analysis and Log of NAN Analysis for Adopted Scheme Over Extant Schemes in Terms of (a) Count of Rounds and (b) Distance.

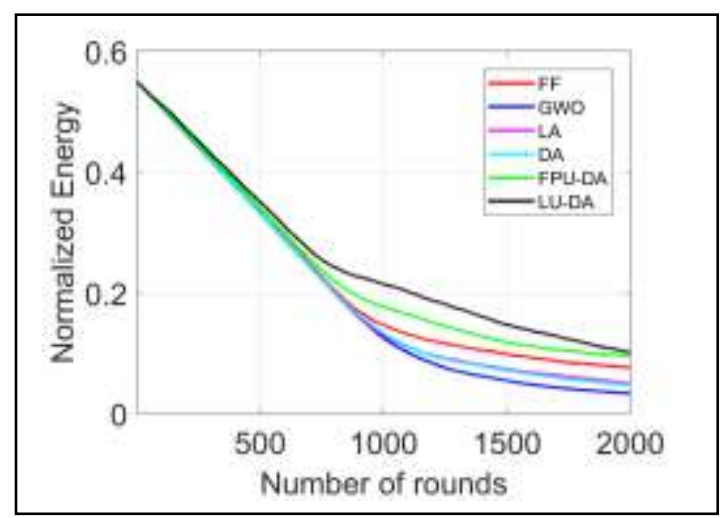

Fig. 4. Analysis on Normalized Energy Attained using Presented Work Over Existing Works.

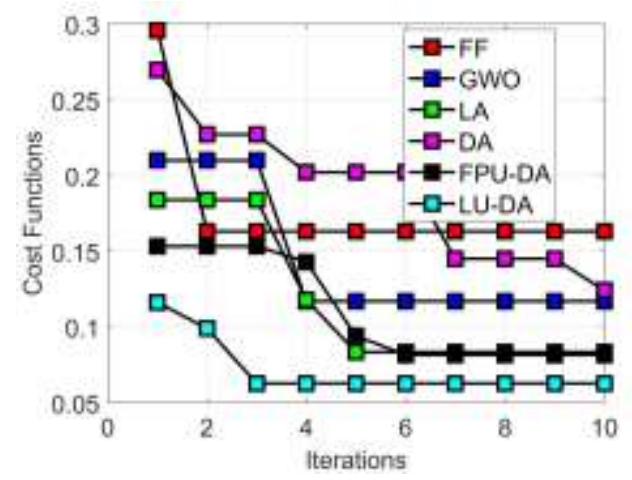

Fig. 5. Convergence Analysis of Developed Scheme Over Traditional Models in Terms of Cost. 


\section{E. Statistical Analysis}

Table III describes the statistical analysis of the presented LU-DA model over prevailing approaches regarding alive nodes, energy, cost and time. "As meta-heuristic schemes are stochastic in nature, every algorithm is executed for the number of times to attain the statistic of objective function". On noticing the resultants, the adopted LU-DA model has obtained high NAN and energy values and minimal cost and time values for all scenarios, when compared over the existing schemes. Especially, on noticing the NAN from Table III, the best case scenario using proposed LU-DA model has attained superior values over distinguished schemes. Moreover, the cost and time values of conventional schemes is superior to the presented model. Nevertheless, at specific scenarios, the conventional schemes have exposed its enhanced performance regarding time. Though, the entire evaluation of objectives reveals the impact of improving the adopted scheme, thus ensuring a secured transmission.

\section{F. Analysis on Delay, Security and Trust}

The analysis on delay, security and trust attained using implemented model over existing models is tabulated in
Table IV. Here, examination is done by altering rounds from 0 to 2000 . On analysing the delay, the presented LU-DA model has obtained high values for trust and minimal values for delay and security (risk) for all rounds. Initially, at round 1, the delay of adopted scheme seems to be 0.98 , while, as the number of rounds increases, the developed method has acquired a minimal delay value of 0.89 at $2000^{\text {th }}$ iteration. Simultaneously, while analysing the security (risk), the developed approach at $1^{\text {st }}$ round has accomplished a minimal risk value of 0.06 , whereas, at $2000^{\text {th }}$ iteration, a comparatively higher risk value of 0.094 has been acquired by adopted model. However, for all rounds, the developed approach has acquired fine outcomes than the compared models as per the desired objectives. Also, while examining the trust values, the adopted method has accomplished a higher trust value of 0.32 at $2000^{\text {th }}$ round, whereas, the compared models like FF, GWO, LA, DA and FPU-DA has acquired relatively minimal values of $0.24443,0.21992$, $0.17411,0.23624$ and 0.23446 . Therefore, the improvement of LU-DA scheme is confirmed from the outcomes.

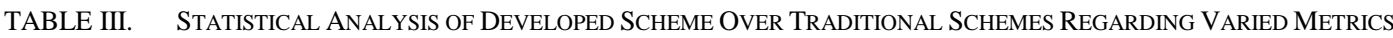

\begin{tabular}{|c|c|c|c|c|c|c|}
\hline \multicolumn{7}{|c|}{ Alive nodes } \\
\hline Measures & $\mathrm{FF}$ & GWO & LA & DA & FPU-DA [24] & LU-DA \\
\hline Mean & 67.887 & 64.314 & 64.819 & 64.82 & 70.774 & 70.693 \\
\hline Best & 30 & 17 & 24 & 22 & 35 & 39 \\
\hline Median & 58 & 65 & 61 & 62 & 61 & 56 \\
\hline Worst & 100 & 100 & 100 & 100 & 100 & 100 \\
\hline STD & 28.791 & 34.082 & 32.67 & 32.304 & 24.449 & 23 \\
\hline \multicolumn{7}{|c|}{ Normalized energy } \\
\hline & $\mathrm{FF}$ & GWO & LA & DA & FPU-DA [24] & LU-DA \\
\hline Mean & 0.21887 & 0.19672 & 0.20528 & 0.20481 & 0.23513 & 0.25519 \\
\hline Best & 0.076316 & 0.033484 & 0.049668 & 0.046585 & 0.095484 & 0.10092 \\
\hline Median & 0.14681 & 0.12504 & 0.13032 & 0.13293 & 0.17645 & 0.21429 \\
\hline Worst & 0.54958 & 0.54958 & 0.54958 & 0.54958 & 0.54958 & 0.54958 \\
\hline STD & 0.14569 & 0.1624 & 0.15523 & 0.15647 & 0.13754 & 0.12921 \\
\hline \multicolumn{7}{|c|}{ Cost Function } \\
\hline & FF & GWO & LA & DA & FPU-DA [24] & LU-DA \\
\hline Median & 0 & 0.14251 & 0.13267 & 0.12611 & 0.11535 & 0.017313 \\
\hline Worst & 0.38671 & 0.25673 & 0.21372 & 0.23847 & 0.19976 & 0.15659 \\
\hline Best & 0.023504 & 0.017313 & 0.029904 & 0.017313 & 0.023528 & 0 \\
\hline Mean & 0 & 0.14035 & 0.1317 & 0.12599 & 0.11456 & 0.023129 \\
\hline STD & 0 & 0.032131 & 0.028361 & 0.031633 & 0.027952 & 0.016704 \\
\hline \multicolumn{7}{|l|}{ Time } \\
\hline & $\mathrm{FF}$ & GWO & LA & DA & FPU-DA [24] & LU-DA \\
\hline Mean & 1.7643 & 1.5862 & 1.4678 & 1.6114 & 4.549 & 2.0993 \\
\hline Best & 1.4428 & 1.4751 & 1.4255 & 1.4872 & 2.6577 & 1.1549 \\
\hline Median & 1.5791 & 1.575 & 1.4588 & 1.5999 & 4.4943 & 1.6068 \\
\hline Worst & 6.16 & 2.0474 & 2.2782 & 6.2107 & 6.888 & 8.0005 \\
\hline STD & 0.58403 & 0.060094 & 0.036912 & 0.12653 & 0.36098 & 1.1177 \\
\hline
\end{tabular}




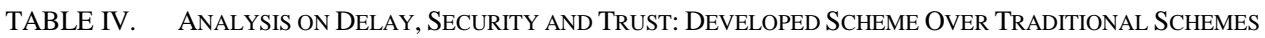

\begin{tabular}{|c|c|c|c|c|c|c|}
\hline \multicolumn{7}{|l|}{ Delay } \\
\hline Rounds & $\mathrm{FF}$ & GWO & LA & DA & FPU-DA [24] & LU-DA \\
\hline 0 & 0.96697 & 0.93975 & 1.0545 & 1.0547 & 0.84699 & 0.98305 \\
\hline 100 & 1.0892 & 1.1839 & 1.1871 & 0.96307 & 1.1825 & 0.92846 \\
\hline 225 & 1.1839 & 0.97482 & 0.94676 & 1.1367 & 1.1019 & 0.92955 \\
\hline 500 & 1.0665 & 1.015 & 1.02 & 1.015 & 1.0442 & 0.93306 \\
\hline 725 & 0.94676 & 0.89849 & 1.1963 & 0.94247 & 1.0012 & 0.89247 \\
\hline 1000 & 1.1112 & 1.0665 & 0.99593 & 1.0291 & 0.98194 & 1.1112 \\
\hline 1225 & 0.89849 & 1.0218 & 1.0545 & 1.0044 & 0.81323 & 1.2899 \\
\hline 1500 & 0.89658 & 0.93068 & 0.9879 & 1.1963 & 0.72301 & 0.94676 \\
\hline 1726 & 1.0119 & 1.015 & 1.02 & 0.97447 & 0.81323 & 1.0218 \\
\hline 2000 & 0.94767 & 1.1644 & 1.0116 & 1.0044 & 0.90933 & 0.893 \\
\hline \multicolumn{7}{|c|}{ Security (risk) } \\
\hline & $\mathrm{FF}$ & GWO & LA & DA & FPU-DA [24] & LU-DA \\
\hline 0 & 0.14793 & 0.11718 & 0.2206 & 0.10757 & 0.077825 & 0.065633 \\
\hline 100 & 0.1713 & 0.18119 & 0.26347 & 0.071713 & 0.12692 & 0.097313 \\
\hline 225 & 0.16429 & 0.15659 & 0.19628 & 0.1246 & 0.10803 & 0.086563 \\
\hline 500 & 0.11718 & 0.10757 & 0.34161 & 0.13545 & 0.090417 & 0.085969 \\
\hline 725 & 0.053169 & 0.15371 & 0.34767 & 0.063057 & 0.018887 & 0.018656 \\
\hline 1000 & 0.089979 & 0.13928 & 0.3868 & 0.14793 & 0.072902 & 0.06253 \\
\hline 1225 & 0.089979 & 0.12584 & 0.22542 & 0.13477 & 0.067928 & 0.065784 \\
\hline 1500 & 0.19505 & 0.13449 & 0.46562 & 0.10825 & 0.077875 & 0.077313 \\
\hline 1726 & 0.090257 & 0.11595 & 0.12679 & 0.14698 & 0.14311 & 0.1272 \\
\hline 2000 & 0.1441 & 0.14451 & 0.19878 & 0.11628 & 0.11925 & 0.094513 \\
\hline \multicolumn{7}{|l|}{ Trust } \\
\hline & $\mathrm{FF}$ & GWO & LA & DA & FPU-DA [24] & LU-DA \\
\hline 0 & 0.25457 & 0.32173 & 0.17335 & 0.34906 & 0.21982 & 0.25919 \\
\hline 100 & 0.28324 & 0.2001 & 0.13852 & 0.25616 & 0.22706 & 0.39341 \\
\hline 225 & 0.21758 & 0.23228 & 0.18336 & 0.21304 & 0.22499 & 0.24245 \\
\hline 500 & 0.32646 & 0.20875 & 0.48212 & 0.33051 & 0.35113 & 0.3607 \\
\hline 725 & 0.21877 & 0.25422 & 0.23838 & 0.25893 & 0.31657 & 0.46922 \\
\hline 1000 & 0.24332 & 0.25835 & 0.19616 & 0.21464 & 0.30112 & 0.4071 \\
\hline 1225 & 0.31622 & 0.20314 & 0.30029 & 0.23106 & 0.24709 & 0.17652 \\
\hline 1500 & 0.26154 & 0.14357 & 0.13765 & 0.38285 & 0.25689 & 0.2413 \\
\hline 1726 & 0.17468 & 0.16548 & 0.29837 & 0.26808 & 0.2644 & 0.51033 \\
\hline 2000 & 0.24443 & 0.21992 & 0.17411 & 0.23624 & 0.23446 & 0.3193 \\
\hline
\end{tabular}

\section{G. Parametric Analysis}

Fig. 6 shows the parametric analysis of normalized energy, convergence, and alive nodes. In the LU-DA scheme algorithm, there is a random number that varies from 0-1. Here, the analysis has been done by varying the random variable $\mathrm{r}=0.2, \mathrm{r}=0.4, \mathrm{r}=0.6, \mathrm{r}=0.8$, and $\mathrm{r}=1$. On observing the results, it can be noticed that when $r=0.4$, the proposed LUDA attains the best results. By setting the random variable $\mathrm{r}=0.4$, the above analysis like normalized energy, convergence, and alive nodes has been portrayed by comparing with other existing models.

\section{H. Discussion}

This paper presents a new LU-DA model for optimal CHS. Here, the optimal $\mathrm{CH}$ selection is carried out by considering the constraints like "energy, delay, distance, security and trust. Here the analysis is performed for alive nodes, normalized energy, convergence analysis, analysis based on delay, security and trust. The betterment of the proposed LU-DA model is proved on various measures like energy and alive node analysis. From the above analysis, it is evident that the proposed model attains better results when compared with the existing models like FF, GWO, LA, DA and FPU-DA. Thereby attaining improved the network lifetime. 


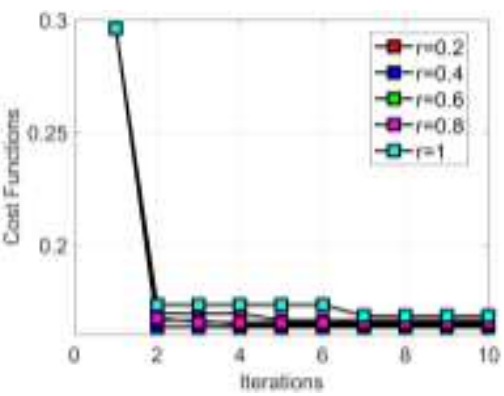

(a)

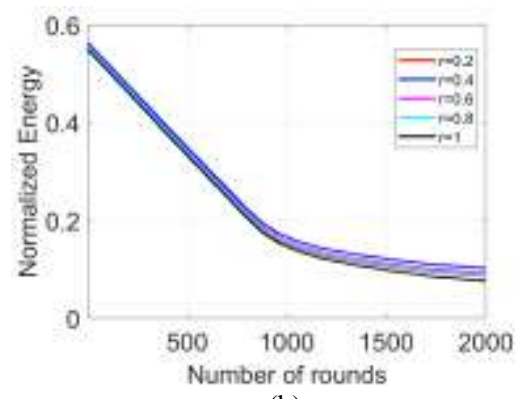

(b)

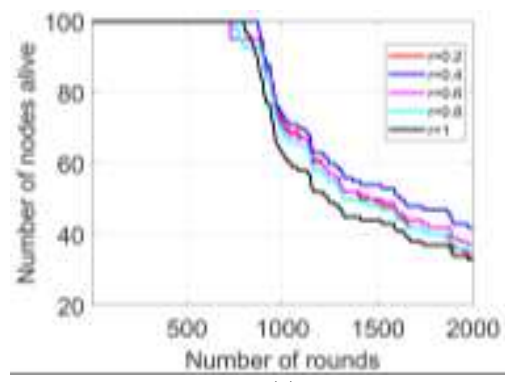

(c)

Fig. 6. Parametric Analysis on (a) Convergence Analysis (b) Normalized Energy (c) Alive Nodes.

\section{CONCLUSION}

This paper introduced a new LU-DA model for optimal CHS. For optimization, a novel approach termed as LU-DA was developed. Eventually, the primacy of offered method was confirmed over conventional models. The presented model has attained higher energy of 0.55 at $1^{\text {st }}$ round, while at the $2000^{\text {th }}$ round; the normalized energy value has been dropped to 0.1. However, the adopted model has accomplished higher energy even at the $2000^{\text {th }}$ round, when distinguished over FF, GWO, LA, DA and FPU-DA models. At the initial iterations (from 1 to 3 ), the cost of the developed model has accomplished a comparatively higher value, while at further iterations, the developed model has converged to a minimal cost value. Especially, at iteration 2, the adopted model was only $60 \%$ enhanced than the extant FF model, while at iteration 10, the adopted model was $62.5 \%$ enhanced than FF model. Therefore, the development of the suggested LU-DA scheme was authenticated over other techniques.

\section{REFERENCES}

[1] Alagumuthukrishnan S, Dr. Geetha K, "A Locality Based Clustering and M-Ant Routing protocol for QoS in Wireless Semsor Networks", Department of science and engineering, vol. 6, no. 10, 14 october 2016.

[2] M. Yuvaraja, Sabrigiriraj M, "Lifetime Enhancement of WSN using Energy-Balanced Distributed Clustering Algorithm with Honey Bee Optimization",Asian Journal of research in social sciences and humanities, vol. 6, no. 11, 2016.

[3] Q. Ni, Q. Pan, H. Du, C. Cao and Y. Zhai, "A Novel Cluster Head Selection Algorithm Based on Fuzzy Clustering and Particle Swarm Optimization," IEEE/ACM Transactions on Computational Biology and Bioinformatics, vol. 14, no. 1, pp. 76-84, 1 Jan.-Feb. 2017.

[4] S. H. Kang and T. Nguyen, "Distance Based Thresholds for Cluster Head Selection in Wireless Sensor Networks," IEEE Communications Letters, vol. 16, no. 9, pp. 1396-1399, September 2012.

[5] J. Leu, T. Chiang, M. Yu and K. Su, "Energy Efficient Clustering Scheme for Prolonging the Lifetime of Wireless Sensor Network With Isolated Nodes," IEEE Communications Letters, vol. 19, no. 2, pp. 259262, Feb. 2015.

[6] Jin Wang, Yiquan Cao, Bin Li, Hye-jin Kim, and Sungyoung Lee, "Particle swarm optimization based clustering algorithm with mobile sink for WSNs, Future Generation Computer Systems, vol. 76, pp 452457, 2017.

[7] Shishupal Kumar, Nidhi Lal, Vijay Kumar Chaurasiya, "A forwarding strategy based on ANFIS in internet-of-things-oriented wireless sensor network (WSN) using a novel fuzzy-based cluster head protocol", Annals of Telecommunications, vol. 73, no. 9-10, pp 627-638, 2018.

[8] Turki Ali Alghamdi, "Parametric analysis on optimized energy-efficient protocol in wireless sensor network", Soft Computing > Issue 6/2021, $20-11-2020$
[9] Turki Ali Alghamdi, "Enhanced QoS routing protocol using maximum flow technique", Computers \& Electrical Engineering, Volume 89, 2021, 106950, ISSN 0045-7906, https://doi.org/10.1016/j.compeleceng.2020.106950.

[10] Turki Ali Alghamdi, "Underwater Wireless Sensor Network Route Optimization using BIHH Technique" International Journal of Advanced Computer Science and Applications(IJACSA), 11(6), 2020. http://dx.doi.org/10.14569/IJACSA.2020.0110645.

[11] Alghamdi, T.A. Route optimization to improve QoS in multi-hop wireless sensor networks. Wireless Netw (2020). https://doi.org/10.1007/s11276-020-02388-y.

[12] D. Jia, H. Zhu, S. Zou and P. Hu, "Dynamic Cluster Head Selection Method for Wireless Sensor Network," IEEE Sensors Journal, vol. 16, no. 8, pp. 2746-2754, April15, 2016.

[13] Pawan Singh Mehra, Mohammad Najmud Doja, and Bashir Alam, "Fuzzy based enhanced cluster head selection (FBECS) for WSN", Journal of King Saud University - Science, Available online, 2018.

[14] Awais, Muhammad \& Ali, Ishtiaq \& Alghamdi, Turki \& Ramzan, Muhammad \& Tahir, Muhammad \& Akbar, Mariam \& Javaid, Nadeem. (2020). Towards Void Hole Alleviation: Enhanced GEographic and Opportunistic Routing Protocols in Harsh Underwater WSNs. IEEE Access. 10.1109/ACCESS.2020.2996367.

[15] Sara Al-Sodairi, and Ridha Ouni, "Reliable and energy-efficient multihop LEACH-based clustering protocol for wireless sensor networks", Sustainable Computing: Informatics and Systems, vol. 20, pp 1-13, 2018.

[16] Gaurav Kumar Nigam, and Chetna Dabas, "ESO-LEACH: PSO based energy efficient clustering in LEACH", Journal of King Saud University - Computer and Information Sciences, Available online, 2018.

[17] R. Raj Priyadarshini, and N. Sivakumar, "Cluster head selection based on Minimum Connected Dominating Set and Bi-Partite inspired methodology for energy conservation in WSNs", Journal of King Saud University - Computer and Information Sciences, Available online, 2018.

[18] Khalid A. Darabkh, Saja M. Odetallah, Zouhair Al-qudah, Ala' F. Khalifeh, and Mohammad M. Shurman, "Energy-Aware and DensityBased Clustering and Relaying Protocol (EA-DB-CRP) for gathering data in wireless sensor networks", Applied Soft Computing, vol. 80, pp 154-166, 2019.

[19] Shilpa Mahajan, Jyoteesh Malhotra, and Sandeep Sharma, "An energy balanced QoS based cluster head selection strategy for WSN", Egyptian Informatics Journal, vol. 15, no. 3, pp 189-199, 2014.

[20] Muthukumaran K, Chitra K, and Selvakumar C, "An energy efficient clustering scheme using multilevel routing for wireless sensor network", Computers \& Electrical Engineering, vol. 69, pp 642-652, 2018.

[21] G. Kannan, and T. Sree Renga Raja, "Energy efficient distributed cluster head scheduling scheme for two tiered wireless sensor network", Egyptian Informatics Journal, vol. 16, no. 2, pp 167-174, 2015.

[22] Palvinder Singh Mann, and Satvir Singh, "Improved metaheuristic based energy-efficient clustering protocol for wireless sensor networks", Engineering Applications of Artificial Intelligence, vol. 57, pp 142-152, 2017. 
[23] M. Marsaline Beno, Valarmathi I. R, Swamy S. M and B. R. Rajakumar, "Threshold prediction for segmenting tumour from brain MRI scans", International Journal of Imaging Systems and Technology, Vol. 24, No. 2, pages 129-137, 2014, DOI: https://doi.org/10.1002/ima.22087.

[24] Alghamdi, T.A. Energy efficient protocol in wireless sensor network: optimized cluster head selection model. Telecommun Syst 74, 331-345 (2020). https://doi.org/10.1007/s11235-020-00659-9.

[25] Prachi MaheshwariAjay K. SharmaKaran Verma, "Energy efficient cluster based routing protocol for WSN using butterfly optimization algorithm and ant colony optimization", Ad Hoc Networks, vol.110 (Cover date: 1 January 2021), Article 102317, 6 October 2020.

[26] Reeta BhardwajDinesh Kumar, "MOFPL: Multi-objective fractional particle lion algorithm for the energy aware routing in the WSN", Pervasive and Mobile Computing, vol. 58, Article 101029, August 2019.

[27] Augustine, S., Ananth, J.P. "Taylor kernel fuzzy C-means clustering algorithm for trust and energy-aware cluster head selection in wireless sensor networks", Wireless Netw 26, pp. 5113-5132, 2020. https://doi.org/10.1007/s11276-020-02352-w.

[28] Pratik Goswami, Ziwei Yan, Amrit Mukherjee, Lixia Yang, Sidheswar Routray, and G. Palai, "An energy efficient clustering using firefly and HML for optical wireless sensor network", Optik, vol. 182, pp 181-185, 2019.

[29] AmanjotSinghToor, A.K.Jain," Energy Aware Cluster Based Multi-hop Energy Efficient Routing Protocol using Multiple Mobile Nodes (MEACBM) in Wireless Sensor Networks", AEU - International Journal of Electronics and Communications, vol.102, pp.41-53, April 2019.

[30] S. M. M. H. Daneshvar, P. Alikhah Ahari Mohajer and S. M. Mazinani, "Energy-Efficient Routing in WSN: A Centralized Cluster-Based
Approach via Grey Wolf Optimizer," in IEEE Access, vol. 7, pp. 170019-170031, 2019, doi: 10.1109/ACCESS.2019.2955993.

[31] WangTianshu, Zhang Gongxuan, YangXichen, VajdiAhmadreza," Genetic algorithm for energy-efficient clustering and routing in wireless sensor networks", Journal of Systems and Software, vol.146, pp.196214, December 2018.

[32] Nejla RouissiHamza GharsellaouiSadok Bouamama, "Improvement of Watermarking-LEACH Algorithm Based on Trust for Wireless Sensor Networks", Procedia Computer Science, vol. 159 (Cover date: 2019), pp. 803-813, 14 October 2019.

[33] Mohammad Jafari and Mohammad Hossein Bayati Chaleshtari, " Using dragonfly algorithm for optimization of orthotropic infinite plates with a quasi-triangular cut-out", European Journal of Mechanics A/Solids, vol. 66, pp.1-14, 2017.

[34] Rajakumar Boothalingam, "Optimization using lion algorithm: a biological inspiration from lion's social behavior", Evolutionary Intelligence, 7 September 2018.

[35] Renjith Thomas and MJS. Rangachar, "Hybrid Optimization based DBN for Face Recognition using Low-Resolution Images", Multimedia Research, Vol.1,No.1, pp.33-43,2018.

[36] Moresh Madhukar Mukhedkar,Uttam Kolekar, "Hybrid PSGWO Algorithm for Trust-Based Secure Routing in MANET", Journal of Networking and Communication Systems, Vol.2,No.3, pp.1-10,2019.

[37] Rupam Gupta Roy, "Rescheduling Based Congestion Management Method Using Hybrid Grey Wolf Optimization - Grasshopper Optimization Algorithm in Power System", Journal of Computational Mechanics, Power System and Control, Vol.2,No.1, pp.9-18,2019. 